\title{
Tsafon
}

Revue d'études juives du Nord

73 | 2017

Connaissance de la Shoah : témoignages, enseignements, visites

\section{Déportations et transferts, un témoignage}

\section{Charles Baron}

\section{(2) OpenEdition \\ Journals}

Édition électronique

URL : https://journals.openedition.org/tsafon/281

DOI : $10.4000 /$ tsafon.281

ISSN : 2609-6420

Éditeur

Association Jean-Marie Delmaire

Édition imprimée

Date de publication : 1 juin 2017

Pagination : 39-46

ISSN : 1149-6630

\section{Référence électronique}

Charles Baron, « Déportations et transferts, un témoignage », Tsafon [En ligne], 73 | 2017, mis en ligne le 31 mai 2018, consulté le 24 juin 2021. URL : http://journals.openedition.org/tsafon/281 ; DOI : https://doi.org/10.4000/tsafon.281 


\section{Déportations et transferts un témoignage}

\section{Charles Baron*}

Je voudrais tout d'abord apporter quelques précisions à ce qui a été dit. Sachez qu'aucun camp n'a été libéré. Les camps ont été découverts au fur et à mesure de l'avance des troupes alliées. En Bavière, où se trouvait le camp de Dachau, les troupes américaines ont découvert des camps à l'odeur! Auschwitz sentait très mauvais aussi, seulement quand vous êtes dans l'atmosphère, vous ne le sentez pas.

Tout à l'heure on a parlé de poésie, on a cité Adorno. Je pense qu'il a dit une sottise. Les poèmes sont très utiles. Un poète, en peu de mots, peut dire beaucoup plus qu'un littérateur en plusieurs pages. Je voudrais citer Pierre Créange ${ }^{1}$ qui était un poète que l'on dit mineur mais

\footnotetext{
* Charles Baron (1926-2016) est décédé le 4 octobre dernier, à l'âge de 90 ans. Dans le $\mathrm{n}^{\circ} 72$ de Tsafon, décembre 2016, Danielle Delmaire lui a rendu hommage. Né à Paris de parents issus de l'immigration d'Europe centrale, Charles Baron fut déporté par le convoi 34, du 18 septembre 1942, deux mois après ses parents, victimes eux de la rafle $\mathrm{du}$ «Vel d'Hiv». Il fit partie des déportés qui furent sélectionnés pour descendre à Kosel, avant d'atteindre Auschwitz. Puis il fut transféré à Dachau. Rapatrié à Paris en juillet 1945, il vécut presque trois années de sa jeunesse dans les camps. Dès les années 1950, il témoigna, dans les écoles, lors de conférences ou dans des colloques, en de nombreuses circonstances, sur les exactions commises par les nazis.

Ce témoignage fut entendu, après celui de Haïm Vidal Sephiha, lors du colloque consacré à l'ouverture des camps, en janvier 2005, à l'université Charles de Gaulle Lille 3. Voir supra. Ici aussi nous avons conservé l'expression orale qui donne l'avantage à l'émotion et aux sentiments du rescapé qui manie volontiers l'ironie.

${ }^{1}$ Pierre Créange (1902-1943) fut déporté, avec sa femme, par le même convoi que Charles Baron. Aucun des deux ne revint. Il était militant de la SFIO, de la LICA et de la Ligue des Droits de l'Homme. (Cette note et toutes les notes suivantes sont de la rédaction).
} 
néanmoins reconnu. Lorsqu'il a voulu décrire le camp et notre situation, moi il m'aurait fallu pas mal de lignes, lui a trouvé un vers extraordinaire, il a parlé de solitude dans la multitude. Et c'était bien vrai. Même si nous étions un ou vingt, on était toujours séparés des autres par quelque chose. Je suis reconnaissant au poète pour cela.

J'ai un dernier compte à vous donner. J'ai eu une chance par rapport à Haïm et par rapport à beaucoup d'autres. Je n'ai jamais fait de marche de la mort. J'ai toujours voyagé en chemin de fer, car j'ai été évacué plus tôt et peut-être aussi parce que j'ai eu un coup de chance.

L'hiver 1944-1945 a été un des plus durs qu'a connu cette guerre. J'ai relevé, dans un document allemand ${ }^{2}$, pour 115 hommes évacués de Dachau vers Auschwitz, c'est-à-dire amenés à la mort, le nombre de vêtements qu'ils possédaient. Les Allemands étaient tellement précis que non seulement ils dressaient les listes des hommes qui partaient mais ce que ceux-ci portaient était aussi noté. Pour 115 hommes, il y avait 115 vestes, 115 pantalons, 86 caleçons - vous vous doutez naturellement dans quel état étaient ces vêtements - aucune paire de chaussettes, 92 chemises, zéro manteaux, 21 pull-over ou ce qui ressemble à un pullover, 38 paires de chaussures de cuir et 57 paires de chaussures en bois et tissus, les illustres claquettes que nous avons tous portées, longtemps. Quand on fait les calculs, on s'aperçoit que parmi ces gens qui sont partis, 20 sont partis pieds nus, sans chaussures, 23 sans chemise, 29 sans caleçon, 94 sans pull over ou similaire, quelques-uns n'avaient pas de chemise, ni de chaussettes, ni de manteau. C'est-à-dire que sur ces 115 hommes qui sont partis de Dachau à Auschwitz, certainement, à mon avis, quelque 30 à $40 \%$ ne sont pas arrivés au bout du voyage. Malheureusement, les archives d'Auschwitz ne donnent pas de détail sur ce sujet. C'était la fin et ils sont arrivés à quelques jours de l'entrée des troupes soviétiques dans le camp, le 27 janvier. Ce jour-là est parti de Dachau un convoi de 10 hommes, ce n'est rien 10 hommes, pour être expédiés, pour mourir, à Auschwitz. On n'a jamais su ce qu'ils étaient devenus. Il n'y a de traces d'eux qu'au départ. C'est tout !

Une autre chose importante. Quand on dit qu'il n'y a jamais eu d'ordre de Hitler pour ériger les chambres à gaz, pour tuer les juifs, c'est vrai ! C'est vrai, Hitler a très peu écrit et c'est ce que déploraient ses

\footnotetext{
${ }^{2}$ Malheureusement, Charles Baron ne nous a pas précisé l'origine de ce document mais comme il s'impliquait dans les actions du Mémorial de la Shoah et comme il avait appartenu au comité de rédaction de la Revue d'Histoire de la Shoah, nous ne mettons pas en doute le sérieux de sa citation.
} 
maréchaux. Lorsqu'une besogne difficile était à accomplir, ils ne recevaient aucune instruction écrite mais arrivait un sémillant SS qui leur disait de faire ceci ou cela. C'est la volonté du Führer.

Le 14 avril 1945, voici la condamnation à mort de 32000 hommes, c'est-à-dire tous les prisonniers du camp de Dachau et des kommandos à l'entour. Ce papier en allemand, je vais me faire le plaisir de vous le traduire, dit : "Au commandant des camps de Dachau et Flossenbourg, la reddition n'est pas en question, les camps doivent être évacués immédiatement». Et voici la phrase qui tue : "Aucun détenu ne doit tomber vivant entre les mains de l'ennemi ». Avec ça, 32000 hommes ont été condamnés à la mort. L'avance des Américains a été beaucoup plus rapide que prévu et nous n'avons pas été exécutés dans les conditions où nous aurions dû l'être. C'est-à-dire, il avait été prévu au départ de nous rassembler sur la place du camp de Dachau, la place d'appel et de nous donner une soupe empoisonnée. Je tiens à la disposition de ceux qui le désirent les références de ces décisions ${ }^{3}$. Ils n'ont pas trouvé assez de poison. Ils ont donc décidé de nous abattre à l'arme à feu. Dans cette période où la destruction des juifs primait sur le ravitaillement des armées allemandes, ils n'avaient pas assez de munitions et ils n'ont pas pu nous tuer.

Ils ont demandé à l'aviation allemande de nous bombarder, une fois rassemblés sur la place d'appel. Là deux versions divergent, actuellement. Les aviateurs disent, avec vertu : " Nous avons dit non, nous ne sommes pas là pour bombarder un camp mais nous sommes là pour faire la guerre ». C'est encore à voir ! Et l'état-major d'infanterie dit: "Lorsque nous avons reçu les instructions, nous avons dit aux aviateurs : ne jouez pas aux couillons, les Américains sont là dans peu de temps, cela risque de nous coûter cher ». En tout cas, toutes ces parlotes ont eu un avantage, c'est que l'on s'en est sortis vivants car ils n'ont pas eu le temps d'accomplir leur besogne. Mais les dépositions qui ont été faites devant les différents tribunaux alliés sont, si vous me permettez le mot, saignantes.

L'essentiel de mon intervention, j'y viens rapidement mais je voulais vous dire tout cela.

Lorsque nous sommes partis de Drancy accompagnés par la police française du camp de Drancy, jusqu'à la gare, on nous a mis dans des

\footnotetext{
${ }^{3}$ Ces références ne nous ont pas été fournies. Voir note 2.
} 
voitures des transports publics de la région parisienne - à l'époque ils s'appelaient la STCRP ${ }^{4}$. Les conditions étaient correctes, il n'y a rien à dire. Quand nous sommes arrivés sur le quai de la gare, là tout a changé. Les wagons étaient de vieux wagons comme on voit dans les films: hommes 40, chevaux en long 8. Simplement, pendant la Guerre de quatorze, ces wagons avaient servi à transporter les troupes. En inscrivant le nombre d'hommes et de chevaux, on comptait plus rapidement les troupes.

Le wagon au sol fait $21 \mathrm{~m}^{2}$. Nous étions, nous les juifs déportés, entre 60 et 80 . Plus proche de 80 que de 60 . Vous voyez tout de suite ce que cela donnait. Un peu de paille sur le sol, un tout petit peu de ravitaillement, rien d'autre. Ah si ! Pardon, deux seaux de 10 litres d'eau. Les seaux étamés qui n'existent plus. Imaginez, pour trois jours, 80 personnes et 20 litres d'eau! Septembre 1942 fut un mois très très chaud. Il y avait avec nous des malades, il y avait des enfants en bas âge, il y avait des vieux. En arrivant à la frontière française de l'époque, c'est-àdire Metz, il n'y avait plus rien à boire. Les seaux étaient vides et ils ont servi de seaux de toilette, parce qu'il ne suffit pas de boire ou de manger le peu de réserves que nous avions, excusez-moi, il faut aussi les évacuer! Il n’y a pas le choix !

Imaginez la scène, à une époque où les mœurs étaient quand même différentes : on n'avait jamais vu une mère faire ses besoins devant ses enfants, un père non plus. On avait mis à notre disposition une couverture de l'armée française, usagée, grise et trouée d'ailleurs. Et lorsque quelqu'un allait faire ses besoins, de chaque côté de la couverture un homme la tenait par un petit bout, pour qu'on ne voie pas grand' chose. On ne voyait pas grand' chose mais l'odeur et les bruits étaient là.

Je veux dire en détaillant cela : comment peut venir à l'idée d'un homme de réduire la dignité des gens à rien, simplement parce qu'on est obligé de faire ses besoins, les uns devant les autres. Les SS n'ont pas hésité. Je me demande ce qui se passait dans la tête des policiers français qui nous amenaient jusqu'au bord du wagon. Ils voyaient bien comment cela se passait, comment on nous entassait, ils avaient vu monter les seaux. Ils n'ont pas eu un moment de réflexion. Tout s'est bien passé !

\footnotetext{
${ }^{4}$ Société des Transports en Commun de la Région Parisienne, elle exploitait les transports des voyageurs dans tout le département de la Seine. En 1942, la STCRP est absorbée par la Compagnie du Chemin de Fer Métropolitain de Paris qui exploitait le métro. À la Libération, la Régie Autonome des Transports Parisiens (RATP) remplaça cette compagnie.
} 
Très rapidement, les seaux pleins ont débordé, sur la paille, sur le fond du wagon. C'était l'horreur absolue.

Mais nous n'avions pas tout vu, car lorsque nous sommes arrivés à Dresde ou à Weimar - je ne me souviens pas, excusez-moi mais à l'époque les guides touristiques manquaient dans les wagons ! - le train s'est arrêté, les portes se sont ouvertes et alors on a pu respirer un peu d'air. Car ces wagons avaient des ouvertures de $80 \mathrm{~cm}$ de hauteur sur $1 \mathrm{~m}$ de large: on ne pouvait pas respirer. L'air nous a fait du bien. Les gardiens allemands ont autorisé deux hommes de corvée par wagon à descendre avec un seau, le seau rempli de déjection, et à le vider. Où ils sont allés le vider, je ne sais pas. Et le comble du luxe et de l'horreur, c'est qu'ils ont dû rincer rapidement les seaux et les remplir avec de l'eau pour que nous ayons quelque chose à boire! Je peux vous dire que dans notre wagon, comme dans les autres wagons, personne n'a bu cette eau souillée.

Si je vous raconte cela, ce n'est pas pour vous raconter une chose horrible, c'est pour vous répéter ce que j'ai dit tout à l'heure. Dans quel esprit malade est venue cette idée de donner à des gens une eau à boire dans laquelle il y avait eu leurs déjections. Où peut-on aller plus loin dans le mépris de l'homme ? Aussi loin, je n'avais pas vu!

Et puis le train a repris son voyage. L'eau des wagons a été évacuée par les interstices du sol des wagons. Et nous avons souffert de la soif jusqu'au moment où le train s'est arrêté dans une campagne, en Silésie, à Kosel. Et là il y avait un SS qui était une espèce de marchand de bestiaux, un négrier. Je ne me souviens plus qu'il ait fait ouvrir la bouche pour voir si nos dents étaient en bon état. En tout cas, c'est lui qui nous a jaugés et a choisi parmi les 1000 déportés, parmi les hommes, peut-être 100 ou 150 hommes pour aller travailler. J'en ai fait partie. Aujourd'hui, je suis revenu et je dis que ce fut une chance. Et je n'en suis pas sûr. En tout cas, nous avons quitté ce train, les autres sont allés à Auschwitz. Et, disons, 80 à $90 \%$ d'entre eux ont été envoyés à la chambre à gaz. Voilà, le voyage aller c'était cela, moi je fus dirigé vers un camp de travail.

Et la Silésie est un pays où il fait gaillardement moins 20 à moins 25 , 1'hiver, à condition que le vent ne s'en mêle pas.

La fin de la guerre, elle, a été un peu différente pour moi. Je n'ai donc pas fait de marche de la mort. J'ai été évacué d'AuschwitzBirkenau le 25 octobre 1944. Nous avons été amenés dans le kommando 
de Dachau, Landsberg. Il était proche d'une petite ville, très jolie d'ailleurs, je l'ai revue il n'y a pas longtemps, je ne l'avais pas vue à l'époque. C'était une ville qui avait pour gloire d'avoir une prison qui avait hébergé Hitler après qu'il a raté son putsch de 1923. C'est vous dire que toute la région était ultra nazie. Lorsque nous avons traversé les petites villes sur des remorques agricoles tirées par des tracteurs qui faisaient un bruit terrible et qui puaient horriblement, les grands-mamans, les bonnes grands-mamans allemandes, étaient au bord de la route avec leurs petits mignons. Les enfants, c'est gentil à 6-7 ans avec les mamans qui étaient là, car les maris étaient quelque part en train de faire la guerre, et les mamans étaient là. Et tout le long du trajet entre la gare de Kosel et mon premier camp dont je me souviens le nom, qui s'appelait Zakrau, c'était un camp de triage, tout le long du trajet, dès qu'on arrivait dans un village, on a trouvé le même comité d'accueil, les grands-mamans, les enfants, les mamans. Les grands-mamans et les mamans engageaient les petits chérubins à nous jeter des pierres, à nous cracher dessus et à nous hurler des injures. Je les entends encore ces injures. Et j'ai retrouvé, il n'y a pas longtemps, un copain qui avait suivi le même chemin : il avait eu le même baptême touristique en Allemagne. Quand on habitue des gosses de cet âge-là à avoir le mépris total de la dignité de l'autre, c'est sûr qu'on arrive aux chambres à gaz d'Auschwitz. C'est direct !

La sortie des camps, j'ai commencé à vous l'expliquer tout à l'heure avec les bombardements. On nous a remis dans des wagons, le temps qu'ils trouvent une solution à nos misères. Notre chance est que nous avons été attaqués par les Américains, les avions américains. On a réussi grâce à un gars qui était un peu plus costaud que les autres à passer par-dessus la rambarde du wagon. C'étaient des minéraliers, sans toit. Il a enlevé les épars et on a ouvert les portes. Nous sommes partis, un médecin parisien et moi, dans la campagne. Je ne sais pas où nous avons trouvé la force de faire des kilomètres et des kilomètres. Nous sommes allés loin avec les SS aux fesses, qui nous recherchaient, mais notre grande chance est qu'ils n'avaient pas de chiens. À un moment, nous sommes entrés dans une espèce de petite forêt, là il y avait une petite cabine de contrôle. Les SS s'étaient arrêtés là et ils étaient perdus, ils ne nous trouvaient plus. S'ils avaient eu des chiens, cela aurait été différent.

Et puis le coup de chance, le coup de chance que n'ont pas eu beaucoup de déportés. Nous avons trouvé un fermier bavarois qui nous a

\footnotetext{
${ }^{5}$ Camp près de Blechhammer qui rassemblait un grand nombre de camps de travail annexes.
} 
proposé, on n'a pas dit non je vous le dis tout de suite, qui nous a proposé de nous cacher en attendant les Américains. D'accord, c'était sur la fin de la guerre mais c'était l'époque où la vie d'un homme ça ne valait rien. Les SS tuaient un homme pour un oui ou pour un non et de temps en temps pour un peut-être. Il n'y avait plus de limites. Il nous a cachés, il a risqué sa vie, celle de sa femme, celle de son fils. Il a risqué qu'on foute le feu à sa ferme, qu'on tue ses bestiaux. Il a vraiment risqué beaucoup pour nous. Et le troisième jour, les Américains sont véritablement arrivés. Nous étions cachés dans un grenier et nous avons vu le ballet des troupes qui se succédaient, les Allemands en retraite, les Américains qui avançaient. Et pour une raison ou pour une autre, les Américains partaient, les Allemands revenaient, enfin ce n'était pas très confortable. Le matin du troisième jour, le fermier est arrivé, tout excité, tout joyeux. Les Américains ! Les Américains ! Il se rendait compte qu'il était à l'abri de la destruction de sa ferme, de la destruction de ses bestiaux. Et c'est comme cela que j'ai vu les premiers soldats américains. Et je dois vous dire que, comme le disent tous ceux qui ont eu la chance de savourer ce moment, c'est inoubliable! Ida Grinspan, ma camarade Ida Grinspan ${ }^{6}$ qui avait quatorze ans quand elle a été déportée, quand on lui demande : « comment c'était ta libération, comment ils étaient les Américains? ». Il faut la voir, elle a les yeux qui roulent puis elle fait : «Ah, ils étaient beaux, ils étaient beaux ! ». Et je crois que j'ai dû les trouver beaux moi aussi.

En tout cas, mon grand souvenir, ça m'a été rappelé par un film qui est passé à la télévision il va y avoir trois ans, Frères d'armes ${ }^{7}$, qui raconte l'histoire d'une unité américaine. J'ai connu les hommes de ces unités, je suis toujours en relation avec eux. Et je me souviens d'une scène de Frères d'armes où on voit un déporté s'accrocher à un militaire

\footnotetext{
${ }^{6}$ Née à Paris, le 19 novembre 1929, Ida Grinspan fut arrêtée dans le Poitou, par trois gendarmes, en janvier 1944, à Lié, hameau de Melle dans les Deux-Sèvres, où elle était réfugiée depuis le printemps 1940. À l'âge de 14 ans, elle fut transférée à Drancy, puis déportée à Auschwitz par le convoi quittant Drancy le 10 février 1944. Ses parents étaient des immigrés polonais mais de nationalité française par déclaration. Sa mère fut victime de la rafle du 16 juillet 1942 et fait partie du convoi 11, du 27 juillet 1942.

Elle témoigne fréquemment dans les établissements scolaires. Elle est l'auteur de $J$ 'ai pas pleuré, en association avec Bertrand Poirot-Delpech, Paris, Laffont, 2002, 2003, 2012.

${ }^{7}$ Frères d'armes est une série télévisée américaine, diffusée en France en 2002 (le témoignage de Charles Baron eut lieu en 2005), de Tom Hanks et Steven Spielberg. Elle comprend dix épisodes d'une heure et retrace l'épopée de divisions américaines depuis leur débarquement en Normandie et leur progression en France et en Europe, jusqu'à l'ouverture des camps.
} 
américain et ne pas vouloir décrocher. J'ai souvent raconté cette scène à ma femme. Quand j'ai vu mon premier soldat américain, j'ai commencé à chialer, et je ne pleure pas souvent, et je me suis accroché à lui. Pour me descendre, il a fait un gros effort, il a pris son temps. Eh bien, j'ai retrouvé cette scène : ça a été un éblouissement ! Et puis après, ils sont repartis de nouveau. On a vraiment été abandonnés par les Américains mais il n'y avait plus d'Allemands. Et dans l'après-midi, sont arrivées les premières troupes qui allaient rester sur place.

Donc ma libération a été un petit conte de fée par rapport à mes copains qui ont fait les marches de la mort. Au fond j'ai eu du pot... un sacré pot!

Voilà, c'est ce que je voulais vous dire. La vie tient entre deux moments, pourvu qu'elle tienne. Dans notre cas, elle a tenu alors je suis bien content.

Le Mémorial de la déportation des juifs de France, édité et publié par Beate et Serge Klarsfeld, 1978, décrit les convois de Charles Baron et d'Ida Grinspan.

Convoi 34 du 18 septembre 1942. Il quitte Drancy avec 1000 juifs.

À l'arrivée de ce convoi à Auschwitz, le 20 septembre, 31 hommes furent gardés en vie avec les matricules 64719 à 64749 . D'autres furent sans doute sélectionnés avant l'arrivée à Kosel... Le reste du convoi fut immédiatement gazé. On comptait en 1945, 21 survivants de ce convoi.

Convoi $68 \mathrm{du} 10$ février $1944^{8}$.

Ce convoi est constitué par 1500 déportés : 674 hommes, 814 femmes et 14 indéterminés. 279 moins de 18 ans..

À l'arrivée à Auschwitz, le 13 février, 210 hommes sélectionnés reçurent les matricules 173708 à 173 917, ainsi que 61 femmes (75 340 à 75 400). 1229 personnes furent aussitôt gazées. En 1945, on comptait 42 survivants, dont 24 femmes.

\footnotetext{
${ }^{8}$ Le nom d'Ida apparaît sur la liste à celui de son père : Fensterzab.
} 